\title{
Initiating a Translational Bio-Mathematics Research Seminar for Undergraduate Students
}

\author{
Turian, Emma ${ }^{a}$ and Filus, Lidia ${ }^{a}$
}

${ }^{a}$ Mathematics Department, Northeastern Illinois University, USA

\begin{abstract}
The aim of this paper is to illustrate the benefits and the drawbacks of an experimental process on how to develop and teach an interdisciplinary applied math course. The analysis comes from our experience gained during the development and teaching of a temporary seminar called: Mathematical Modeling for Cancer Risk Assessment, implemented at our University. The need for the initiation of such an interdisciplinary course came from an increasing national effort started by Mathematical Association of America's "Curriculum Foundations Project: Voices of the Partner Disciplines". Their study found that research in biology and health-related fields has become more quantitatively oriented than in the past, therefore mathematical curricula should incorporate interdisciplinary modulation. Our seminar instruction included: writing and mathematical software skills, content lecture, project development and presentation. Results showed that students best interact with each other if work is performed during class time; mainly if a large project with possible variations is developed in class, so students or groups of students follow using the same pace. Implementing such interdisciplinary course that provided students with appropriate tools and methodologies, contributed to student retention, and increased students' enthusiasm towards future research programs, carriers, and graduate schools.
\end{abstract}

Keywords: cancer modeling; interdisciplinary fields; multidisciplinary instruction. 


\section{Introduction}

The need for the formation of the Mathematical Modeling for Cancer Risk Assessment seminar came from an increasing national effort started by MAA's "The Curriculum Foundations Project: Voices of the Partner Disciplines" (MAA 2004), to make undergraduate courses more compelling and keep pace with the changes of how mathematical sciences are used. Based on views expressed during a series of disciplinary workshops, MAA completed an extensive review which resulted in a set of recommendations for the Undergraduate Programs in Mathematics to assist math departments plan their curriculums in the 21st century. These workshops consisted of dialogs among representatives from the partner discipline with mathematicians. A final Curriculum Foundation Conference resulted in a Collective Vision, which consists of a set of recommendations for the first two years of the undergraduate mathematical curriculum. Together the reports and the Collective Vision, became the pillars for the Committee's Guide (CUPM 2004) for the Undergraduate Program in Mathematics, and later on for the 2015 Guide, Schumacher (2015). The agreement that prompted the need for such a "reform" in the undergraduate curriculum, came from the general consensus that the research in biology and health-related fields has become more quantitatively oriented than in the past. While diverse, these quantitative needs require innovative solutions, such as creation of mathematical courses designed specifically for the bio-medical fields, or mathematical modules that could become incorporated into existing bio-medical courses. The experimental creation of our seminar was an example of the former, and attracted a large audience of students. The goal was to provide students with a platform for jumpstarting their research career. The curriculum of this course included teaching writing and scientific software, learning how to analyze data, make inferences and predictions that are meaningful to one important branch of this bio-medical field, named cancer research. The seminar was specifically designed to enhance students' preparedness for careers, which apply quantitative methodology to government organization, industries, education, biomedical fields and research involving complex behavior.

\section{Methods}

The seminar taught during Spring 2017 became a collaboratively taught research environment that provided a way to incorporate interdisciplinary instruction, where students used mathematics in the context of an application from the Bio-Mathematical field, and it offered a unique opportunity for students to apply their mathematical knowledge, become exposed to research, develop presentation skills, publish their work and present at domestic and international conferences. 
At the end of the course students were required to produce a paper-like research project and to present their findings at a conference. The final grade was based on in-class quizzes (20\%), homework assignments (30\%), a paper-like project (30\%), and an oral presentation or poster $(20 \%)$. The system included alternative grading for projects with considerable mathematical content.

Instruction for this course included four main components as follow:

\subsection{Teaching the usage of a TeX editor}

At first students were required to download a TeX version on their laptop, specifically using the MiKTeX or MacTeX distribution. Successive lectures included showing students how to perform fundamental tasks in LaTeX, such as the use the maketitle command, create table of contents, sections, tables, insert pictures, references, citations, flowcharts using PGF/TikZ, and how to use the begin/end environments for Theorems, Lemmas, equations, lists, symbols, i.e. OEIS (2017), ShareLaTeX (2018), etc. In order to easily transfer work created in LaTeX to presentation slides, students were shown how to use Beamer, which allowed for the creation of flexible and professional looking presentations, i.e. ShareLaTeX (2018) and Wright (2017). Homework assignments were asking students to create documents from a provided template for their editor. Since students were learning TeX writing for producing paper-like documents, and oral presentations, templates for creating a TikZ poster were developed, i.e. ShareLaTeX (2018). Learning how to write papers, develop oral presentation slides, and posters completed the LaTeX cycle.

\subsection{Teaching Mathematica}

Since students were not required to purchase a textbook, they were encouraged to acquire Mathematica software. Features of this software were displayed during class using live board projections while students were using their computer. Basic features of continuous modeling using Mathematica were shown such as: define a function, solve an equation, plot a function, solve an ordinary differential equation analytically and numerically, display data along a given function, and save plots so they can later be inserted into TeX documents. Future lectures also included discrete modeling functionality such as graphs, data organization; statistical components such as Pearson Correlation coefficient, tests for normality, AIC, AICc, BIC, confidence intervals, etc.; and optimization. The main Mathematica functionality that students enjoyed through exercise was the Manipulate function, which gave them instant clues regarding bifurcation parametrization.

The important lesson learned from this was that students learn best if all the Mathematica functionality was placed into context. As such, homework assignments increasingly included more real-life problems that were later needed for their projects. In class quizzes were open notes/books and laptops. 


\subsection{Teaching cancer vocabulary and existing mathematical modeling related to cancer}

Cancer vocabulary and existing mathematical modeling was next introduced to the students using a lecture like format. The sequencing of the lecture presentations was as follow:

1. Categorize existing mathematical modeling related to cancer. Cancer vocabulary, a history of mathematical modeling and current tendencies were presented to the students from Araujo (2004) and Cristini \& Lowengrub (2010). The material was divided into cancer growth (in-vitro) and cancer regression during treatments. The latter was also divided into different categories of treatment (i.e. radiation therapy, chemotherapy, immunotherapy) and the mathematics that is used in the existing literature for each one of them was explained on the board.

2. Analyze the content of published papers. Published results were presented and analyzed during lecture. Key points of existing papers were analyzed and features of each were described and debated (i.e. what an abstract, an introduction, ..., conclusions section should include). Lectures included methodology for distinguishing between a) the biological and b) mathematical problem from a provided publication.

Students were tested on the ability to recognize specifications related to the mathematical and biological aspect from the modeling problem from a given published article (i.e. Figure 1. Sample student questionnaire).

\subsection{Create a paper-like project}

The biggest challenge for having to teach this research course was that students had different mathematical backgrounds. The most sensible decision was to have students choose their projects based on their mathematical background. The drawback to such approach was that in time, students required assistance and it became challenging for the instructor to respond to all requests during class time, and therefore difficult for them to advance their research.

All students completed a paper-like project to the best of their ability. Project feedback helped students to later create professional posters (most of them in LaTeX). 
1. Is this a continuum, discrete or hybrid model?

2. Is this a tumor growth or tumor reduction under therapy model?

3. The problem in this work represents a:

- Darcy Model

- Stokes Model

- Darcy-Stokes Model

- None of these

4. Based on the space dimension, this study is a:

- 2D model

- 3D model

5. Based on the state of tumor and media, is this a:

- avascular - in vitro

- vascularized tumor modeled in silico or in vivo

- angiogenesis - in silico

6. Based on the number of phases, is this a:

- single-phase model

- multi-phase model

7. Based on the mathematical tool, this is $a(n)$ :

- ODEs problem - to model tumor as a homogeneous population

- PDEs problem, which infludes the diffusion equation

- Integro-differential eq. problem, showing the evolution of heterogeneous cells.

8. What is (are) the main finding(s). Describe (i.e. include terms such as parameter meaning, tumor morphology, tumor evolution).

Figure 1. Sample student questionnaire.

\section{Conclusions and future implementation}

Northeastern Illinois University has a rich tradition of educational innovation and research is starting to emerge as an important component of its educational activities. Therefore, for such course, in order to prove successful, educational details such as "time-space" optimization techniques had to be implemented to ensure its success. Time optimization relates to the time spent on each of the teaching objectives, which in our case was about one month for the 2.1 and 2.2 objectives, 3 weeks for objective 2.3, and about 2 months for objective 2.4. This time optimization scheme proved to be beneficial for the most of the course participants, but not for those who had to switch to a different project. For the latter category a larger amount of time for the 2.4 objective seemed to be needed. By "space" optimization we refer to the type of teaching objectives as well as space configuration schemes for grouping students during the development of their projects. Students were grouped according to their mathematical background and a leader for each group was 
selected. Since larger groups of students who shared materials were more productive, having one project with different components (i.e. same computational scheme but different mathematical functionality to reflect tumor dynamic) for the entire class might seem to be a more efficient solution.

The most successful and the least successful implementations, along with proposed new strategies were as follow:

- $\quad$ Most successful teaching strategies:

○ TeX instruction

○ Mathematica instruction

- $\quad$ Least successful teaching methods and proposed replacement strategies:

- Implementing criteria on how to read and later structure a research paper, i.e. Borja (2014), and Weingast (2010), seemed like a good idea. Analyzing the components of a published article in class, followed by proper evaluation criteria improved student comprehension, however increasing the amount of time spent on such objective might seem beneficial to students.

- Having different groups working on different projects. Overseeing different projects from different groups of students might prove exhausting for the instructor and unstable for the students, since they may wish to migrate from one research group to another and possible socialize while the instructor is taken by a group different from the one where the discussion is taking place. Having all students work on the same or similar project, and therefore use similar Mathematica functionality, but perhaps different equations might prove a good replacement strategy. Students could later choose to explore different project ramifications.

- Interdisciplinary collaboration. In our case, the collaboration consisted of some students being able to collaborate with familiar faculty from biology. This could benefit some students but not the entire class. One improvement may include extending invitations to give talks to specialists from the industry, improve collaboration with faculty from biology who worked on related projects, and/or former students. Also, by 
pairing up biology major students with students from the mathematics department might improve student performance.

Students from Mathematics, Computer Sciences, Biology, Chemistry, Environmental Sciences, and Education took this course, which proved to be a dynamic and energizing experience for them as well as for the instructor. The seminar provided students with the opportunity to learn applied mathematics in a context that students find compatible with their interests. The seminar also enriched instructor's experience regarding teaching a research course. The effectiveness of this particular activity was assessed using a survey and the results showed that out of all the responders that wish to continue their education, $75 \%$ agreed and $25 \%$ strongly agreed that the course influenced their decision to continue their education. Of these, $50 \%$ are considering a career in a bio-medical field. Also, from the responders about one third expect to use LaTeX in the near future, one third expect to use Mathematica software and one third expect to continue cancer research. As far as course improvements is concerned, about one third would have liked the course to include additional theoretical background and $20 \%$ would have liked that the course includes more medical terminology. The seminar concluded with student poster presentations at the International Conference on Risk Analysis (ICRA7). Eight posters were presented by our students. Of these, one poster won first prize, NEIU (2017). Based on positive feedback from faculty and students as well as student success, our institution agreed to permanently include this course in our university's curriculum.

This experience has taught us that in order to be successful in such an endeavor the instruction should include the right tools (such as TeX writing and Mathematica software), and the desire to maintain the correct balance between the computational and theoretical understanding of the subject matter. Proper research standards should be maintained and additional real-life projects could be included.

\section{References}

Araujo, R. P., \& McElwain, L. S. (2004). A History of the Study of Solid Tumour Growth: The Contribution of Mathematical Modelling. Bulletin of Mathematical Biology, 66, 1039-1091.

Borja, A. (2014). 11 steps to structuring a science paper editor will take seriously. Elsevier. Retrieved from: https://www.elsevier.com/connect/11-steps-to-structuring-a-sciencepaper-editors-will-take-seriously (accessed: February 5, 2018)

Cristini, V., \& Lowengrub, J. (2010). Multiscale Modeling of Cancer: An Integrated Experimental and Mathematical Modeling Approach. U.K. Cambridge. 
Marc U-Star Program at NEIU. Retrieved from: http://cs.neiu.edu/wordpress/index.php/conferences-2/ (accessed: April 29, 2018)

OEIS. (2017). List of LaTeX mathematical symbols. Retrieved from: https://oeis.org/wiki/List_of_LaTeX_mathematical_symbols (accessed: February 4, 2018)

ShareLaTeX. Beamer. (2018). Retrieved from: https://www.sharelatex.com/learn/Beamer (accessed: February 4, 2018)

ShareLaTeX. Posters. (2018). Retrieved from: https://www.sharelatex.com/learn/Posters (accessed: February 4, 2018)

Schumacher, C. S., \& Siegel M. J. (2015). 2015 CUPM Curriculum Guide to Majors in the Mathematical Sciences. MAA. Retrieved https://www.maa.org/sites/default/files/pdf/CUPM/pdf/CUPMguide_print.pdf (accessed: February 7, 2018)

Weingast, B. R. (2010). CALTECH RULES FOR WRITING PAPERS: * HOW TO STRUCTURE YOUR PAPER AND WRITE AN INTRODUCTION. Retrieved from: https://web.stanford.edu/group/mcnollgast/cgi-bin/wordpress/wpcontent/uploads/2013/10/CALTECH.RUL_..pdf (accessed: April 12, 2018)

Wright, J. (2017). CTAN. The BEAMER class. User Guide for version 3.50. Retrieved from: $\quad$ http://tug.ctan.org/macros/latex/contrib/beamer/doc/beameruserguide.pdf (accessed: April 29, 2018) 